\title{
Jogo Conhecendo o Espaço do Museu de Oceanografia: utilizando interface natural no processo de ensino aprendizagem
}

\author{
Bastos ML', Marques PCF ${ }^{2}$, Dantas MWG ${ }^{1}$, Silva $\mathbf{L N}^{3}$, Silva $\mathrm{JS}^{4}$ \\ ${ }^{1}$ Bacharelado em Sistemas de Informação - Universidade Federal Rural de Pernambuco \\ (UFRPE) \\ Caixa Postal 063 - CEP 56909-535 - Serra Talhada - PE - Brasil \\ ${ }^{2}$ Departamento de Estatística e Informática - DEINFO - Universidade Federal Rural de \\ Pernambuco (UFRPE) \\ Recife - PE - Brasil \\ ${ }^{3}$ Departamento de Sistemas de Informação - Faculdade Estácio - Recife - PE - Brasil \\ ${ }^{4}$ Departamento de Engenharia de Pesca - Universidade Federal Rural de Pernambuco \\ (UFRPE) \\ Recife - PE - Brasil \\ \{michaellb17, pcfmarques.bsi, mayawgd, luzianeenunes\} @gmail.com, \\ jacque_ss@hotmail.com
}

\begin{abstract}
Natural Interaction can be a good alternative to diversify the way content is transmitted. To make the user interact more directly with the subject, and through challenges instigate him to want to know more and more, allows the teaching of the spontaneous way, promoting a better engagement with a subject of difficult transmission. The game Conhecendo o Espaço was created aiming to provide the user curiosities about the planets of our Solar System through a playful environment and with natural interactivity, which is done through the use of the Microsoft Kinect sensor. The project as a whole was carried out using an experimental process where activities of Topic Identification, Information Gathering, Game Design, Interface Design, Implementation and Validation were performed consecutively. All the evaluations of the validation process were extremely positive, from the monitors of the Museu de Oceanografia to the submission phase of the final report, and only for future work the testing of the target audience of the game.
\end{abstract}

Resumo. A Interação Natural pode ser uma boa alternativa para diversificar a forma como os conteúdos são transmitidos. Fazer com que o usuário interaja de maneira mais direta com o assunto, e através de desafios instigá-lo a querer conhecer cada vez mais, possibilita o ensino de maneira espontânea, promovendo um melhor engajamento com um assunto de difícil transmissão. Visando isso, o jogo Conhecendo o Espaço foi criado. A aplicação tem como objetivo fornecer ao usuário curiosidades sobre os planetas do nosso Sistema Solar, através de um ambiente lúdico e com interatividade natural, que é feita através do uso do 
VI Congresso Brasileiro de Informática na Educação (CBIE 2017)

Anais do XXVIII Simpósio Brasileiro de Informática na Educação (SBIE 2017)

sensor Microsoft Kinect. O projeto como um todo foi realizado utilizando um processo experimental onde foram executadas atividades de Identificação de Tema, Levantamento de Informações, Game Design, Projeto de Interface, Implementação e Validação de maneira consecutiva. Todas as avaliações do processo de validação foram extremamente positivas, desde os monitores do Museu de Oceanografia até a fase de submissão do relatório final, ficando apenas para trabalhos futuros a realização de testes com o público-alvo do jogo.

\section{Introdução}

Desde o final da última década, métodos naturais de interação humano-computador estão se popularizando. Isso tem acontecido devido ao surgimento e expansão de sensores de detecção de movimento que começaram a ser largamente utilizados por consoles famosos de video games. Observando esse crescimento, várias áreas do conhecimento visam utilizar da melhor maneira possível os benefícios que tais tecnologias são capazes de trazer, e a educação é uma delas [Aguiar e Battatiola 2010].

A utilização dessas novas atividades proporcionadas pelas tecnologias atuais, são coisas bastante discutidas na educação hoje em dia. Pois, as mesmas não são apenas métodos pedagógicos que relacionam um conteúdo com a utilização de desenhos, imagens, sons ou apenas animações, essas novas técnicas podem ser consideradas como métodos de mediação e expressão, onde a partir delas pode ser possível a criação de novos meios para construir conhecimento [Santos et al. 2014].

Com o intuito de diversificar a forma como os conteúdos são transmitidos, usar a interação natural pode ser uma boa alternativa. Fazer com que o usuário interaja de maneira mais direta com o assunto, e através de desafios, instigá-lo a querer conhecer cada vez mais, possibilita o ensino de maneira espontânea, proporcionando assim, um melhor engajamento com um assunto que pode ser difícil de transmitir [Ferreira 2014].

Com base nessas inovações, o jogo Conhecendo o Espaço foi desenvolvido e é aplicação que faz parte do acervo do Museu de Oceanografia da Unidade Acadêmica de Serra Talhada (MO-UAST). O objetivo é fornecer ao usuário informações sobre os planetas do nosso Sistema Solar, através de um ambiente lúdico e promover uma interatividade natural entre o cenário e o jogador.

\section{Metodologia}

Para o desenvolvimento da aplicação, utilizou-se alguns recursos tecnológicos e pedagógicos. O projeto como um todo foi realizado utilizando um processo experimental, onde a primeira etapa foi nomeada de Identificação Temática, pois foi realizada uma sondagem dentro do próprio Museu, a respeito de qual tema deveria ser abordado naquele momento para melhor se encaixar às necessidades do $\mathrm{MO}$, já que o mesmo possui alguns jogos voltados a diversas temáticas dentro do contexto da Oceanografia.

A segunda etapa foi denominada Levantamento de Informações, pois apesar de ser um tema relativamente simples, as informações transmitidas devem ser atrativas, e para isso buscou-se curiosidades a respeito do tema, com a finalidade de estimular o 
VI Congresso Brasileiro de Informática na Educação (CBIE 2017)

Anais do XXVIII Simpósio Brasileiro de Informática na Educação (SBIE 2017)

usuário a se interessar pelo conteúdo ao longo da aplicação.

A terceira etapa foi para a criação do Game Design. As características gerais do jogo foram desenvolvidas, o modo de interação, cenário, pontuação, recompensa, regras e desafio. Esse é um ponto muito importante do desenvolvimento, pois é através dele que é definido se um jogo é interessante ou não para o fim que está sendo proposto.

$\mathrm{Na}$ quarta etapa, foi realizado o Projeto de Interface, onde os elementos gráficos foram criados, especificamente personagens, backgrounds, indicadores de pontuação e objetos (medalhas, asteroides, etc) que foram desenhados e vetorizados em um plano 2D. A relação entre as cores nesse processo foi essencial, pois isso irá interferir diretamente na relação do usuário com aplicação, e quanto mais atrativa e agradável for essa interface, mais cômodo o jogador vai se sentir ao jogar.

As duas últimas etapas do projeto foram a Implementação e Validação, onde a primeira é simplesmente colocar em prática o que foi definido nas etapas descritas anteriormente, transformando e unindo as ideias e materiais desenvolvidos em código, e a segunda foi feita juntamente com os membros e a supervisora geral do Museu, onde cada um deu sua avaliação técnica e opinião a respeito do que foi desenvolvido. Todas as etapas desse processo de construção estão resumidas na Figura 1.

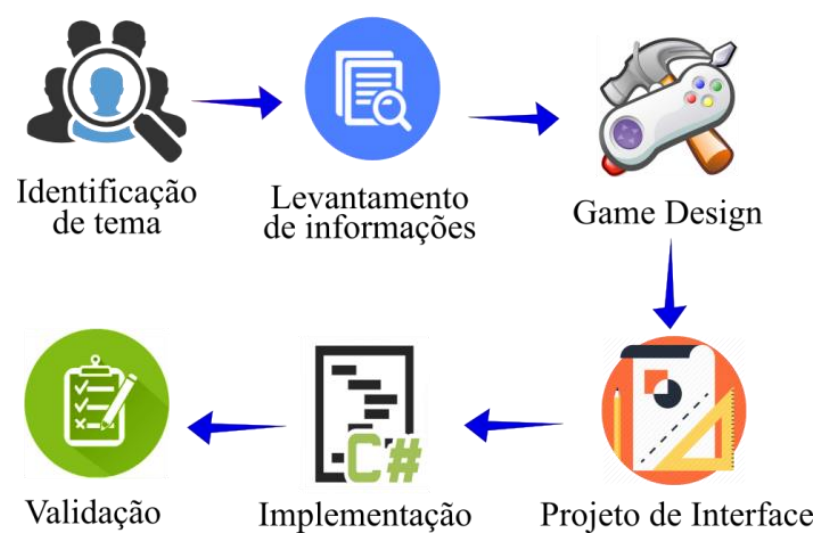

Figura 1. Etapas do processo de desenvolvimento

Algumas tecnologias foram utilizadas para o desenvolvimento do projeto. Para a codificação foi usada a linguagem de programação $\mathrm{C} \#$, utilizada juntamente com a $\mathrm{IDE}^{1}$ de desenvolvimento Visual Studio 2010. A aplicação foi criada para o uso com o sensor Microsoft Kinect, devido a isso, foi necessário a utilização do framework de desenvolvimento Microsoft XNA, para melhor utilizar os recursos proporcionados pela ferramenta.

\section{Fundamentação Teórica}

No mundo computacional, existem várias maneiras diferentes utilizadas para enviar

\footnotetext{
${ }^{1} \mathrm{IDE}$, é um ambiente utilizado para facilitar a programação em um determinado tipo de linguagem de programação.
} 
informações ao computador, e a forma que o homem utiliza para se comunicar com os sistemas computacionais são chamados de Interação Humano Computador (IHC), que é em sua maioria dominante feita através de mouses, teclados e joysticks. Todavia, novas formas de interação estão sendo amplamente expandidas, e se tornando cada vez mais comuns[Ferreira 2014].

Um dos campos da IHC que vem ganhando destaque nos últimos anos é a Interação Natural (IN), pois como o próprio nome já diz, busca interpretar informações naturais passadas pelo usuário e através delas fornecer ações para o computador executar, sem a necessidade do uso de aparelhos físicos, interagindo de forma espontânea, como se fosse um ambiente real[Ferreira 2014].

Complementarmente as técnicas já mencionadas, o uso de jogos pode ser uma opção a mais para aumentar o interesse do usuário pela aplicação. Há algum tempo vem sendo estudada a inserção de jogos em contextos como educação, treinamento e até mesmo reabilitação de pacientes na Medicina, por estar diretamente relacionado com a necessidade contínua de atingir objetivos, envolvendo fatores competitivos e instigar sempre o usuário a se manter motivado[Aranha et al. 2014].

No que se diz respeito ao âmbito educacional, o índice de aceitação e interação do aprendiz com as atividades didáticas digitais, está estritamente ligado ao quão amigável a interface é para o aluno. Assim, pode-se dizer que o uso de interfaces naturais instiga ainda mais o processo de ensino aprendizado do usuário, pois a mesma diminui o quanto é preciso saber sobre como utilizar, concentrando ainda mais o foco no conteúdo que está sendo repassado. Por conseguinte, utilizar os benefícios dos jogos aliados aos benefícios da IN, pode ser uma ótima ferramenta para tornar o aprendizado mais simples e interessante [Santos et al. 2014].

Neste contexto, existem diversas áreas que podem usufruir desses novos modelos de ensino, e a Astronomia é uma delas. Apesar de ser uma ciência bastante antiga, ela também é muito dinâmica, e está sempre se atualizando devido ao avanço tecnológico dos grandes telescópios, sondas e satélites. Mudanças como a quantidade de planetas do nosso Sistema Solar só foram possíveis de descobrir graças a esses avanços concedidos por essas novas tecnologias [Rodrigues 2007].

Os conceitos relativos à Astronomia são bastante discutidos há muitos anos, tanto em Ciências, como em Geografia e no mundo da Física. Uma vasta quantidade de publicações e pesquisas também começaram a ser voltadas especificamente para o público infantil, com a publicação de algumas revistas específicas para facilitar o entendimento das crianças a respeito desse assunto, trazendo discussões a respeito do Sistema Solar, das estrelas e várias curiosidades de como essa ciência veio evoluindo até os dias atuais [Rodrigues 2007].

\section{O jogo Conhecendo o Espaço}

O jogo Conhecendo o Espaço é uma aplicação com novos recursos para o setor de Jogos Digitais do MO. O jogo se passa no espaço, mais especificamente no nosso sistema solar, e o personagem principal, o astronauta $\mathrm{MO}$, deve se esquivar de uma chuva de meteoros que um pequeno extraterrestre (ET) provoca de sua nave. 
VI Congresso Brasileiro de Informática na Educação (CBIE 2017)

Anais do XXVIII Simpósio Brasileiro de Informática na Educação (SBIE 2017)

Juntamente com os meteoros, o ET também envia algumas medalhas, as quais devem ser coletadas pelo astronauta para que possa seguir firme na sua conquista ao espaço. Essas medalhas são demonstradas na Figura 2.
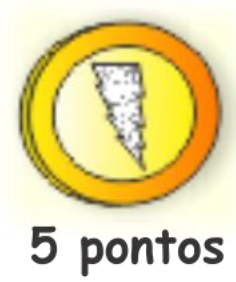
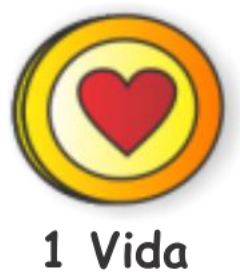
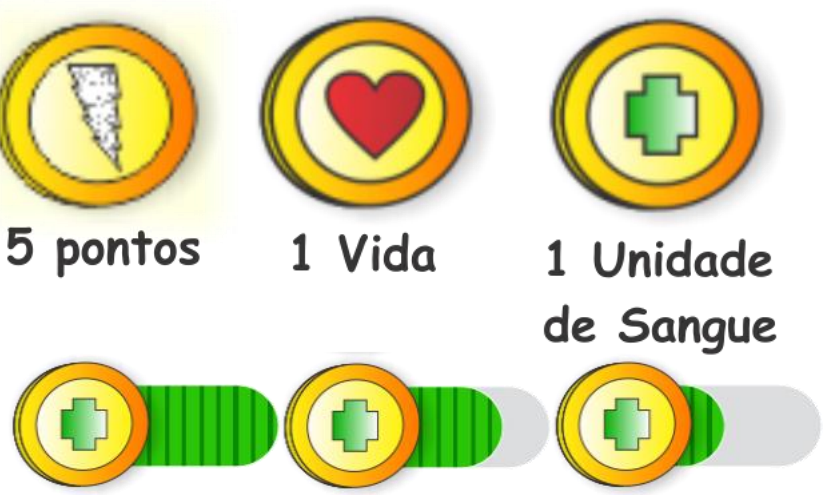

Figura 2. Representação das medalhas do jogo e do medidor de sangue

A medalha com o símbolo de um raio é a que corresponde à pontuação, e vale 5 pontos. A medalha com um coração, tem valor de uma vida a mais para o jogador. $\mathrm{E}$ por fim, a medalha de "sangue" com o símbolo da cruz verde, a cada unidade coletada aumenta um pouco a sobrevida do jogador.

Para que o jogador possa evoluir, ele deve conseguir coletar as medalhas com símbolo de raio e não ser destruído pelos meteoros, que aumentam de velocidade e quantidade ao longo do tempo, dificultando a vida do jogador. O tempo também é um diferencial, pois tudo deve ser feito em um intervalo de 100 segundos por fase do jogo.

A pontuação exigida para conhecer cada planeta é explicada a seguir:

- 20 pontos: O jogador chega ao planeta Mercúrio;

- 50 pontos: O jogador chega ao planeta Vênus;

- 80 pontos: O jogador chega ao planeta Terra;

- 110 pontos: O jogador chega ao planeta Marte;

- 140 pontos: O jogador chega ao planeta Júpiter;

- 170 pontos: O jogador chega a Saturno;

- 200 pontos: O jogador chega a Urano;

- 230 pontos: O jogador chega a Netuno e finaliza o jogo.

A cada transição, uma animação aparece com a imagem do astronauta $\mathrm{MO}$, a pontuação ganha, e algumas curiosidades sobre os planetas que estão sendo conquistados. Uma dessas imagens está representada a seguir (Figura 3A), juntamente com o cenário principal (Figura 3B), e a Tela Inicial da aplicação (Figura 3C), respectivamente, destacando planetas, astros e os demais componentes do jogo. 
VI Congresso Brasileiro de Informática na Educação (CBIE 2017)

Anais do XXVIII Simpósio Brasileiro de Informática na Educação (SBIE 2017)

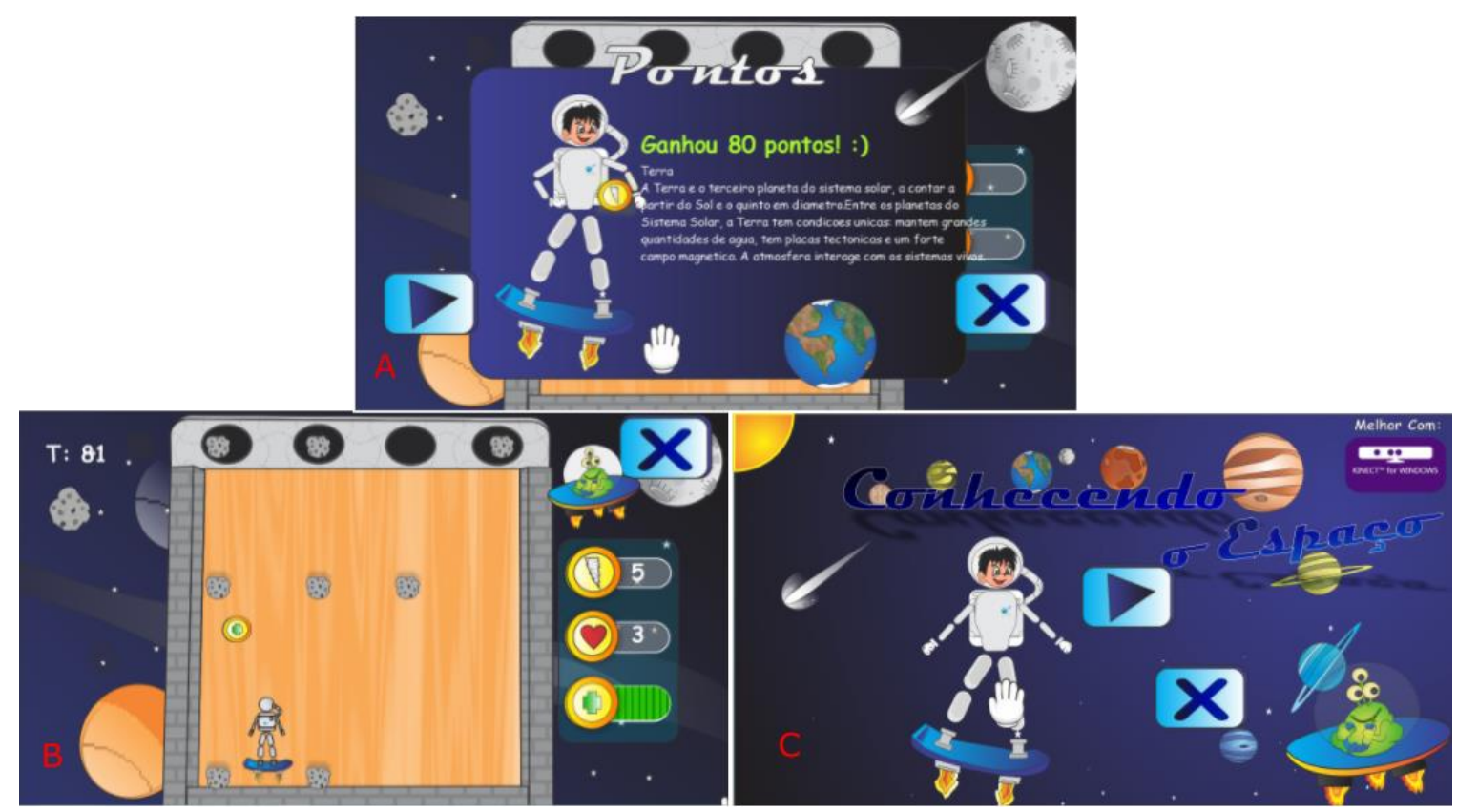

Figura 3. Tela de conquista ao atingir 80 pontos e chegada ao planeta Terra $(A)$, Cenário do jogo (B) e Tela Inicial (C), respectivamente.

Todas as imagens do jogo foram criadas ou também vetorizadas a partir de outras, sendo realizadas modificações em sua estética para diferenciá-las das imagens utilizadas como base. Algumas ilustrações criadas no projeto de interface da aplicação estão demonstradas na Figura 4.

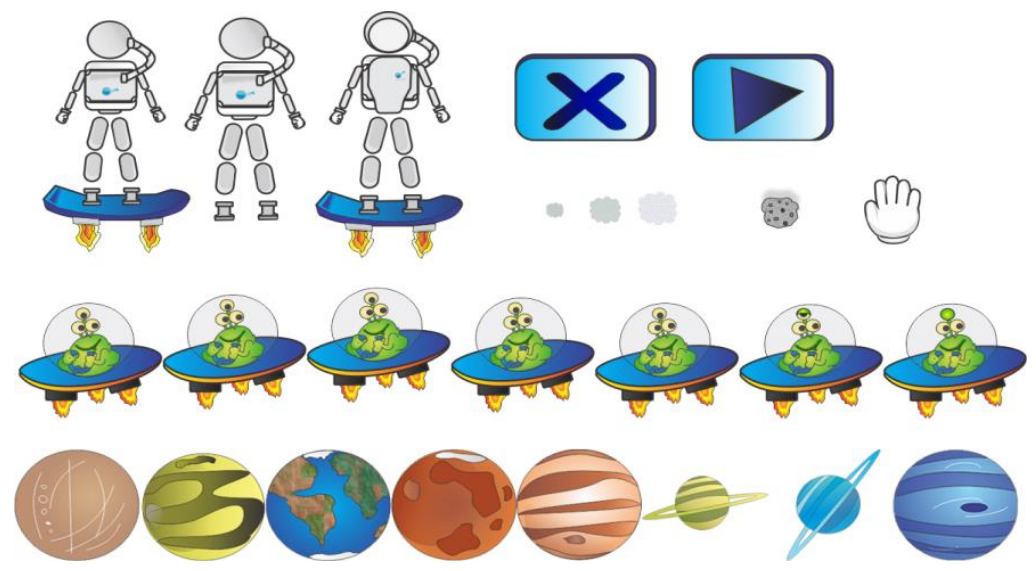

Figura 4. Uma parte do Projeto de Interface do jogo

A aplicação foi construída para ser melhor utilizada com o sensor Microsoft Kinect, fazendo com que além de propagação de conhecimento a respeito dos planetas do nosso Sistema Solar, haja a promoção de atividades aeróbicas para cada jogador, tendo em vista que ele deverá se movimentar para se esquivar da chuva de meteoros e coletar cada medalha. Mesmo sendo criada especificamente para o Kinect, a aplicação pode ser utilizada sem o mesmo. 
VI Congresso Brasileiro de Informática na Educação (CBIE 2017)

Anais do XXVIII Simpósio Brasileiro de Informática na Educação (SBIE 2017)

\section{Trabalhos correlatos}

Santos et al. (2014) apresenta em seu trabalho a aplicação GeoEdu, uma ferramenta voltada para educação, que assim como o Conhecendo o Espaço, também usufrui das contribuições derivadas da interação natural para auxiliar o processo de ensino aprendizagem. O software concentra seus objetivos no ensino de Geografia, o mesmo tem a premissa possibilitar a interação do usuário através de gestos manuais, e com isso instigar o aluno usuário a conhecer suas diversas habilidades diferentes. Para a validação do sistema, foi feito um estudo de caso em ambiente real com a finalidade de analisar o quão impactante pode ser a inserção de uma aplicação assim, no âmbito educacional, tendo como resultado a comprovação da evolução do conhecimento dos jogadores ao longo do uso da aplicação.

Pedrosa et al. (2015) desenvolveu um jogo educativo voltado para crianças de 4 a 6 anos, também utilizando da interatividade promovida pelo Microsoft Kinect. $\mathrm{O}$ mesmo tem como objetivo trabalhar o pensamento e a lógica matemática através dos princípios de ensino piagetiano para o ensino de matemática na pré-escola. O jogo possui vários desafios, e em cada um deles busca a resolução de uma problemática diferente, buscando sempre instigar o usuário a resolver os desafios proposto de maneira lúdica e divertida. Todavia, o autor não chegou a realizar testes com o públicoalvo do trabalho, explicitando sua realização em trabalhos futuros.

Outro uso que vem se popularizando muito, é o uso de interface natural para inclusão e melhoria do ensino de pessoas com algum tipo de necessidade especial. Rodrigues et al. (2015) mostra em seu trabalho o software educacional Abcê Bulir, que é um jogo sério criado com o intuito de ajudar docentes no processo inicial de alfabetização de crianças e adolescentes com Síndrome de Down (SD). A aplicação possui uma série de módulos que foram definidos e concebidos com base em um conjunto de requisitos previamente definidos, que foram obtidos através de pesquisa bibliográficas a respeito do processo cognitivo de crianças com Síndrome de Down. O jogo foi exposto a alguns testes, e segundo o autor, o Abcê Bulir proporcionou um quociente de aprendizagem de aproximadamente $57 \%$, estando dentro das expectativas e comprovando o bom enquadramento da interação natural para com pessoas com SD.

Contudo, vendo o uso da Interação Natural dando certo em vários segmentos distintos da educação, é louvável concentrar esforços na tentativa de ampliar ainda mais essa gama de novas tecnologias para o ensino pedagógico, sem contar na imersão que tais tecnologias proporcionam, deixando o ensino prazeroso, e fazendo as pessoas e principalmente as crianças perceberem que é possível aprender e se divertir ao mesmo tempo, desmistificando o pressuposto de que aprender é uma tarefa exaustiva do nosso dia-a-dia.

Fazendo um comparativo do presente trabalho com os outros aqui relacionados, podemos encontrar o principal diferencial relacionado a maneira com que a temática é abordada. O conhecendo o espaço tem o intuito de trazer para crianças do sertão uma nova forma de conhecimento, relacionando um jogo interativo a uma temática de grande importância para esses alunos. Ele se torna um jogo que induz ao conhecimento de maneira espontânea, e não apenas uma tentativa passar algum tipo de conhecimento através de um jogo. 


\section{Validação}

O processo de validação da aplicação foi feito através de processos distintos. O foco dessa etapa foi na qualidade do jogo e do conteúdo pedagógico fornecido pelo mesmo, e não no modo de interação e usabilidade fornecida pela aplicação, pois isso é algo a ser feito na fase de testes com o usuário, que são trabalhos futuros.

Sendo assim, a primeira a validação foi realizada juntamente com os Monitores do MO, alunos de cursos como Ciências Biológicas e Engenharia de Pesca avaliaram o conteúdo das informações contidas no jogo e através das experiências obtidas pelos mesmos nas diversas exposições do Museu, indicaram que tipo de informações seriam mais adequadas para serem repassadas às crianças que visitam o acervo, já que muitas vêm de regiões muito carentes, onde o acesso à informação e a educação é bastante limitado. No projeto também haviam alunos de Sistemas de Informação, os quais ficaram encarregados de fazer a avaliação da parte técnica do jogo, imagens, interface gráfica e até mesmo da parte relacionada a Interação Humano-Computador.

A segunda etapa da validação foi feita juntamente com a coordenadora do projeto, a qual fez a solicitação do desenvolvimento da aplicação. Com ela, foram feitas análises a respeito da viabilidade e qualidade da aplicação para com o acervo do Museu de Oceanografia, já que o setor de jogos digitais estava precisando de tecnologias que pudessem trazer uma interatividade maior entre os visitantes e o acervo já existente.

Por fim, foi realizada a submissão do jogo para ser aprovada pelo órgão financiador do projeto, a Fundação do Amparo a Ciências e Tecnologia de Pernambuco (FACEPE), onde foi devidamente avaliado e aceito.

Todas avaliações do processo de validação foram extremamente positivas, desde os monitores até a fase de submissão do relatório final. É importante ressaltar que essa fase foi de extrema importância, pois o jogo passou pelas mãos de profissionais e futuros profissionais qualificados, que puderam identificar características a serem ajustadas e/ou a serem adicionadas para tornar o conteúdo da aplicação o mais adequado possível para seu público-alvo, que são as crianças de 8 a 12 anos, visitantes do acervo do Museu de Oceanografia da UFRPE/UAST.

\section{Considerações Finais}

A oceanografia é uma ciência que está relacionada a diversas outras ciências, como geografia, história, química, física e também a astronomia, questão até o momento pouco abordada.

Os métodos pedagógicos estão cada vez mais se inovando e procurando maneiras de fixar conteúdo da melhor maneira possível para os alunos. Realizar movimentos naturais para executar uma atividade lúdica e ainda junto a isso aprender conteúdos muitas vezes exaustivos e difíceis de ser aprendidos, são incentivos grandes no momento de escolher uma ferramenta de ensino, unindo facilidade e entretenimento em um único lugar.

O jogo Conhecendo o Espaço, foi criado com o intuito principal de unir 
exatamente esses dois fatores supracitados, facilidade e entretenimento na hora de aprender. Provendo um ambiente lúdico, curiosidades a respeito da temática geral e uma nova forma de interação com o usuário, o público pode unir o útil ao agradável, em um mesmo ambiente.

Pretende-se com o jogo, que os usuários exercitem e absorvam o máximo de informações possíveis sobre os planetas do Sistema Solar que são demonstradas a cada etapa da aplicação. Porém, devido a uma pausa temporária nas atividades do Museu, a aplicação ainda não participou des exposições no acervo. Mas como trabalho futuro, está a realização de testes para verificar a aceitação da proposta da aplicação por crianças de uma faixa etária de 8 a 12 anos, e avaliar o quanto de informações dos conteúdos sobre a Astronomia foi possível absorver pelos usuários.

\section{Referências}

Aguiar, M. P. e Battatiola, A. L. (2010) "Proposta de um instrumento de auxílio ao design de jogos eletrônicos educativos", Universidade Federal do Paraná, LAI-DI UFPR Laboratório de Animação e Interação e Design da Informação, Brasil.

Aranha, R. V., Ribeiro, M. W. S. e Camargo, C. A. X. de. (2014) "Serious Games e Interação Natural na Terapêutica Posterior ao Tratamento do Câncer de Mama", SBC - Proceedings of SBGames, Culture Track, XIII SBGames, Porto Alegre, RS, Brazil.

Ferreira, D. S. (2014) "Interação Natural Por Meio de Gestos para Apoio a Docentes no Processo de Ensino em Saúde",Universidade Federal Da Paraíba, Centro De Informática, Departamento de Informática, João Pessoa, Brasil.

Pedrosa, D. C., Notargiacomo, P. e Lopes, P. B. (2015) “Jogo Educativo Pré-escolar com Interface NUI para Ensino", Nuevas Ideas en Informática Educativa TISE, Santiago, Chile.

Rodrigues, M. A. (2007) "Os Planetas Do Sistema Solar Em Livros Didáticos De Ciências Da Quinta Série Do Ensino Fundamental ", Experiências em Ensino de Ciências - V2(2), pp. 01-10, Universidade Federal de Pernambuco, Centro de Educação, Departamento de Métodos e Técnicas de Ensino, Recife, Brasil.

Rodrigues, M. S. (2015) "Utilização de Interface Natural de Usuário no Processo de Alfabetização e Desenvolvimento Cognitivo de Crianças com Síndrome de Down", Universidade Federal Rural De Pernambuco Unidade Acadêmica De Serra Talhada Bacharelado Em Sistemas De Informação, Serra Talhada, Pernambuco, Brasil.

Santos, C. P., Segatto, R., Teixeira, Y. P. e Willers, T. F. (2014) "Interface Natural na Educação: Um Estudo de Caso no Ensino de Geografia", $3^{\circ}$ Congresso Brasileiro de Informática na Educação (CBIE), 20ª Workshop de Informática na Escola (WIE), Brasil. 